\title{
Genome-wide DNA methylation profiling is able to identify prefibrotic PMF cases at risk for progression to myelofibrosis
}

\author{
Ulrich Lehmann ${ }^{*}$ (0), Helge Stark, Stephan Bartels, Jerome Schlue, Guntram Büsche and Hans Kreipe
}

\begin{abstract}
Background: Patients suffering from the BCR-ABL1-negative myeloproliferative disease prefibrotic primary myelofibrosis (pre-PMF) have a certain risk for progression to myelofibrosis. Accurate risk estimation for this fibrotic progression is of prognostic importance and clinically relevant. Commonly applied risk scores are based on clinical, cytogenetic, and genetic data but do not include epigenetic modifications. Therefore, we evaluated the assessment of genome-wide DNA methylation patterns for their ability to predict fibrotic progression in PMF patients.

Results: For this purpose, the DNA methylation profile was analyzed genome-wide in a training set of 22 bone marrow trephines from patients with either fibrotic progression $(n=12)$ or stable disease over several years $(n=10)$ using the $850 \mathrm{k}$ EPIC array from Illumina. The DNA methylation classifier constructed from this data set was validated in an independently measured test set of additional 11 bone marrow trephines ( 7 with stable disease, 4 with fibrotic progress). Hierarchical clustering of methylation $\beta$-values and linear discriminant classification yielded very good discrimination between both patient groups. By gene ontology analysis, the most differentially methylated CpG sites are primarily associated with genes involved in cell-cell and cell-matrix interactions.
\end{abstract}

Conclusions: In conclusion, we could show that genome-wide DNA methylation profiling of bone marrow trephines is feasible under routine diagnostic conditions and, more importantly, is able to predict fibrotic progression in prefibrotic primary myelofibrosis with high accuracy.

Keywords: DNA methylation, 850 k EPIC array, Myelofibrosis, Prefibrotic PMF

\section{Introduction}

Primary myelofibrosis (PMF) belongs to the $B C R / A B L 1$ negative myeloproliferative neoplasms and is often characterized by a bi-phasic course of disease. In the initial phase, the disease manifests itself with thrombocytosis and granulocytosis without bone marrow fibrosis $[1,2]$. This phase can last for years (stable disease). In a considerable subset of cases, progression takes place leading to bone marrow fibrosis, extramedullary hematopoiesis with splenomegaly, and frequently thrombocytopenia.

*Correspondence: Lehmann.Ulrich@MH-Hannover.de Institute of Pathology, Medical School Hannover, Medizinische

Hochschule Hannover, Carl-Neuberg-Str. 1, 30625 Hannover, Germany
The duration of stable disease and the risk to fibrotic progression varies substantially between individual patients [3]. Progressive bone marrow fibrosis is a life-threatening condition and, hematopoietic stem cell transplantation (HSCT) provides the only curative therapy. Considering the considerable risk connected with HSCT $[4,5]$, the proper identification of patients with higher risk of fibrotic progression is of utmost importance.

So far the identification of molecular risk factors with overall survival or blastic transformation as endpoints has focused on karyotype, gene expression, and genetic alterations [6-8]. Recently, we have analyzed the association of fibrotic progression in PMF cases with age-related clonal hematopoiesis $(\mathrm{ARCH})$ [9]. Another age-related 
phenomenon in hematopoietic cells is the alteration of epigenetic patterns, mainly gene methylation [10]. Surprisingly little is known about the role of epigenetic aberrations in this context. DNA methylation, the best known and best understood epigenetic mechanism which is altered in many pathological conditions [11, 12], is analyzed only in a few studies about MPNs. Some early studies employing in part now outdated methodology analyzed the DNA methylation of only single genes [1315]. More comprehensive approaches employing Illumina's $27 \mathrm{k}$ array or $450 \mathrm{k}$ array methodology focused on the differences between MPN patients and healthy controls or the differences between MPN subtypes [16-18]. None of these studies addressed the relationship between epigenetic alterations and progression from a pre-fibrotic stage to overt myelofibrosis.

Therefore, we analyzed the DNA methylation profile in bone marrow trephines from patients with pre-fibrotic PMF at diagnosis with no indications for excess of blasts or marrow fibrosis (EB0 and MF0) who later developed myelofibrosis (MF2 or 3). The results were compared with the DNA methylation patterns in bone marrow trephines from prefibrotic PMF patients who showed stable disease for at least four years after diagnosis. For this purpose, the most recent version of the Illumina DNA methylation array was employed ( $850 \mathrm{k}$ EPIC array).

\section{Results}

\section{Suitability of routinely processed FFPE bone marrow} trephines for genome-wide DNA methylation analyses

In a first step, we analyzed 4 samples using the Illumina EPIC array in order to figure out whether the amount and quality of genomic DNA extracted from the routinely processed, fixed, decalcified, and embedded bone marrow trephines in our institution provide a reliable and evaluable signal output.

Altogether, 658,746 out of 865,859 CpG sites were evaluable (see "Material and Methods" section for details). That means, only $14 \%$ of the CpG under showed a signal to noise ratio too low for proper evaluation, demonstrating the feasibility of EPIC DNA methylation array analyses of FFPE bone marrow trephines. This offers the unique opportunity to combine molecular studies with histomorphological examination. Other groups could demonstrate this for different tissue types as well [19-21].

The amount of genomic DNA used for DNA methylation analysis and the age of the sample (both are important variables under routine conditions and for archival specimens) could be excluded as confounding factors in our series of altogether 33 samples (using the Singular Value Decomposition algorithm in ChAMP and a $p$-value cutoff of 0.05 [22], see Additional file 1: Figure S1).

\section{Genome-wide DNA methylation profiling identifies patients suffering from fibrotic progression}

In order to establish a DNA methylation classifier for fibrotic progression, a training set of 22 samples (10 displaying stable disease, 12 fibrotic progression) was used (for inclusion criteria see Materials and Methods section). A test set of additional 11 samples (7 displaying stable disease, 4 fibrotic progression) was used in a second step for independent confirmation of the methylation classifier.

Unsupervised hierarchical clustering of the 1000 most differentially methylated $\mathrm{CpG}$ sites yielded a very good separation of both patient groups. Figure 1 shows the heatmap for the 25 most differentially methylated CpG sites for the combined training and test set. Patients displaying fibrotic progression are clearly separated from the patients with stable disease. Since all samples were taken from patients in the hypercellular early pre-fibrotic phase dominated by granulopoiesis and megakaryopoiesis the cellular composition, which is later changing over the course of the disease and may influence DNA methylation patterns, can be excluded as confounding factor. Additional file 2: Figure S2 displays the clustering for the training and the test set separately and Additional file 3: Figure S3 displays the results for the 1000 most differentially methylated regions.

\section{Identification of a minimal set of differentially methylated CpG sites}

Further analysis of the differentially methylated CpG sites revealed that smaller subsets could be used to discriminate samples displaying fibrotic progress from patients with stable disease, the minimum being $6 \mathrm{CpG}$ sites (Fig. 2a, see also Additional file 4: Figure S4 for $100 \%$ separation of the SD and FP cohort in the training and test set separately based on only these $6 \mathrm{CpG}$ sites). In the next step, linear discriminant analysis (LDA) [23] was performed using the above described training set off 22 samples and the minimal subset of $6 \mathrm{CpG}$ sites. The resulting classifier was subsequently validated against 11 independently measured samples ( 7 with stable disease, 4 with fibrotic progress) and yielded very good accuracy against both training and test set. Figure $2 \mathrm{~b}$ shows the perfect discrimination between the two groups.

Table 1 lists the exact localization and the functional annotation of the $6 \mathrm{CpG}$ sites which are required and sufficient for discrimination of the two patient groups as well as their coefficients for the linear discriminant model. Four of the six CpG sites are associated with known genes, coding for transcription factors as well as proteins involved in cell division and cytokine signaling. 


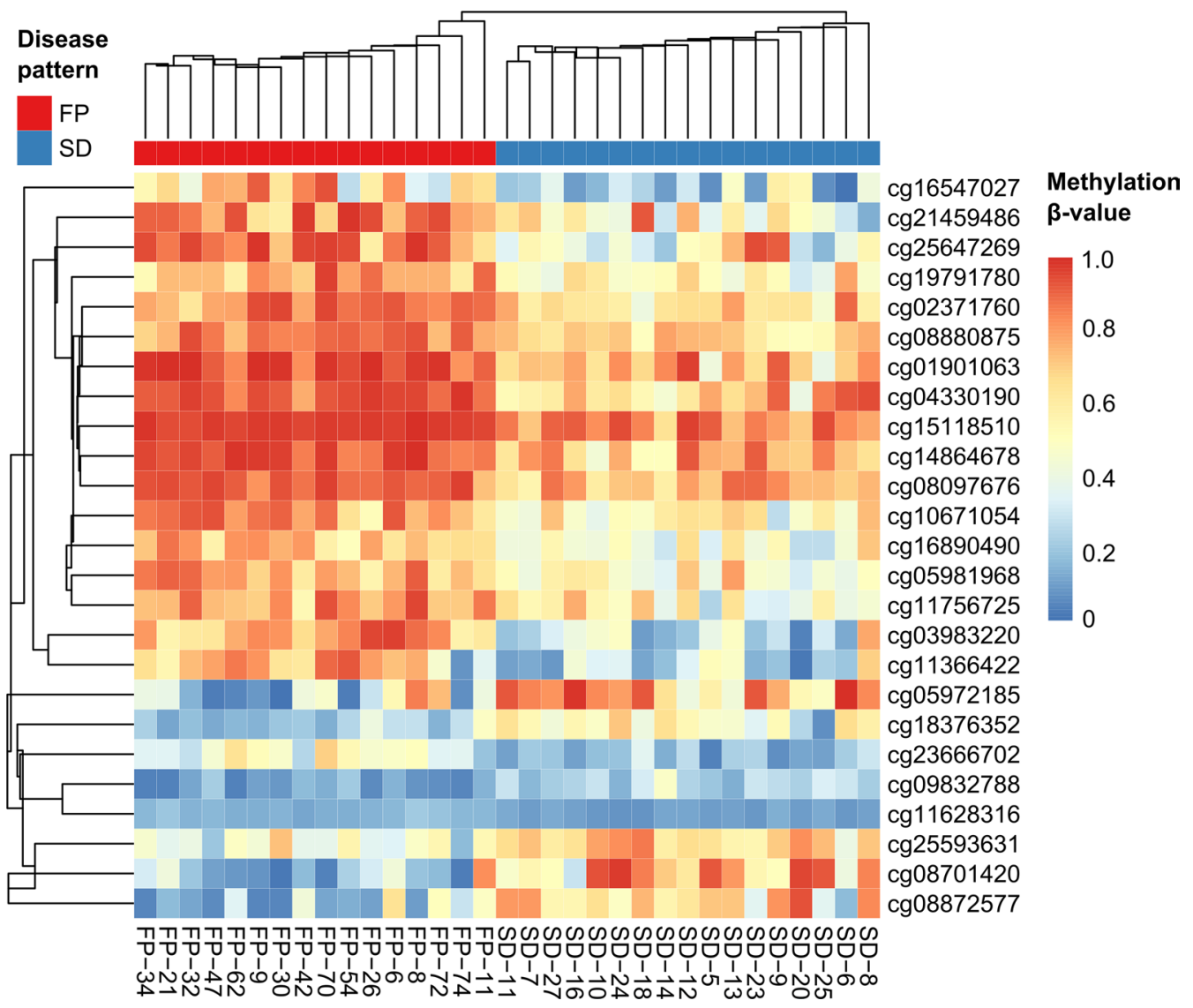

Fig. 1 Hierarchical clustering heatmap of differentially methylated probes. Centroid clustering was used as linkage criterion. Shown are all significantly differentially methylated probes between samples with fibrotic progression (FP) and those with stable disease (SD) at an FDR threshold of 0.1. Additional file 12: Table S3 contains the exact location of all $25 \mathrm{CpG}$ sites. Additional file 3: Figure S3 shows the hierarchical clustering for training and test cohort separately.

A gene set enrichment analysis against the Gene ontology (GO) database based on the 25 most differentially methylated probes yielded no statistically relevant results. Therefore, we used differentially methylated regions as input instead, which revealed a striking enrichment of the biological functions "cell-cell and cell-matrix adhesion" (see Additional file 5: Table S1 and Additional file 6: Table S2).

\section{Analysis of suboptimal samples}

Initially, 7 samples (out of 40) failed the stringent quality metrics evaluation process. Three out of these 7 samples missed the thresholds of various parameters only minimally, thereby representing suboptimal samples which can be encountered under routine diagnostic conditions.

Additional file 7: Figure S5 demonstrates that these 3 samples are correctly classified if the LDA classification algorithm established with the training and test set is applied. These promising results should be confirmed with more suboptimal samples for further demonstration of the usefulness of our newly developed DNA methylation classifier for routine diagnostic analyses.

\section{Hypo- and hypermethylation in stable disease versus fibrotic progression}

The heat maps in Figs. 1 and 2a) already indicate by visual inspection that fibrotic progression is associated with hypermethylation ( $\beta$-values with red color code) relative to the samples from patients with stable disease. A more detailed analysis of the differentially methylated CpG sites confirms this visual impression: The mean $\beta$-value ( \pm standard error) of all 25 methylated CpG sites in the fibrotic progression group is $0.64 \pm 0.02$, versus $0.52 \pm 0.01$ in the stable disease group $(p=1.3 \mathrm{e}-12$, Mann-Whitney- $U$ test). Additional file 8: Figure S6 illustrates in a more global view the gain in hypermethylated loci $(\beta$-value $>0.8)$ in the cohort showing fibrotic progression compared to the group with stable disease. Additional file 9: Figure S7 and Additional file 10: Figure S8 shows this in more detail (Additional file 9: Figure S7) 


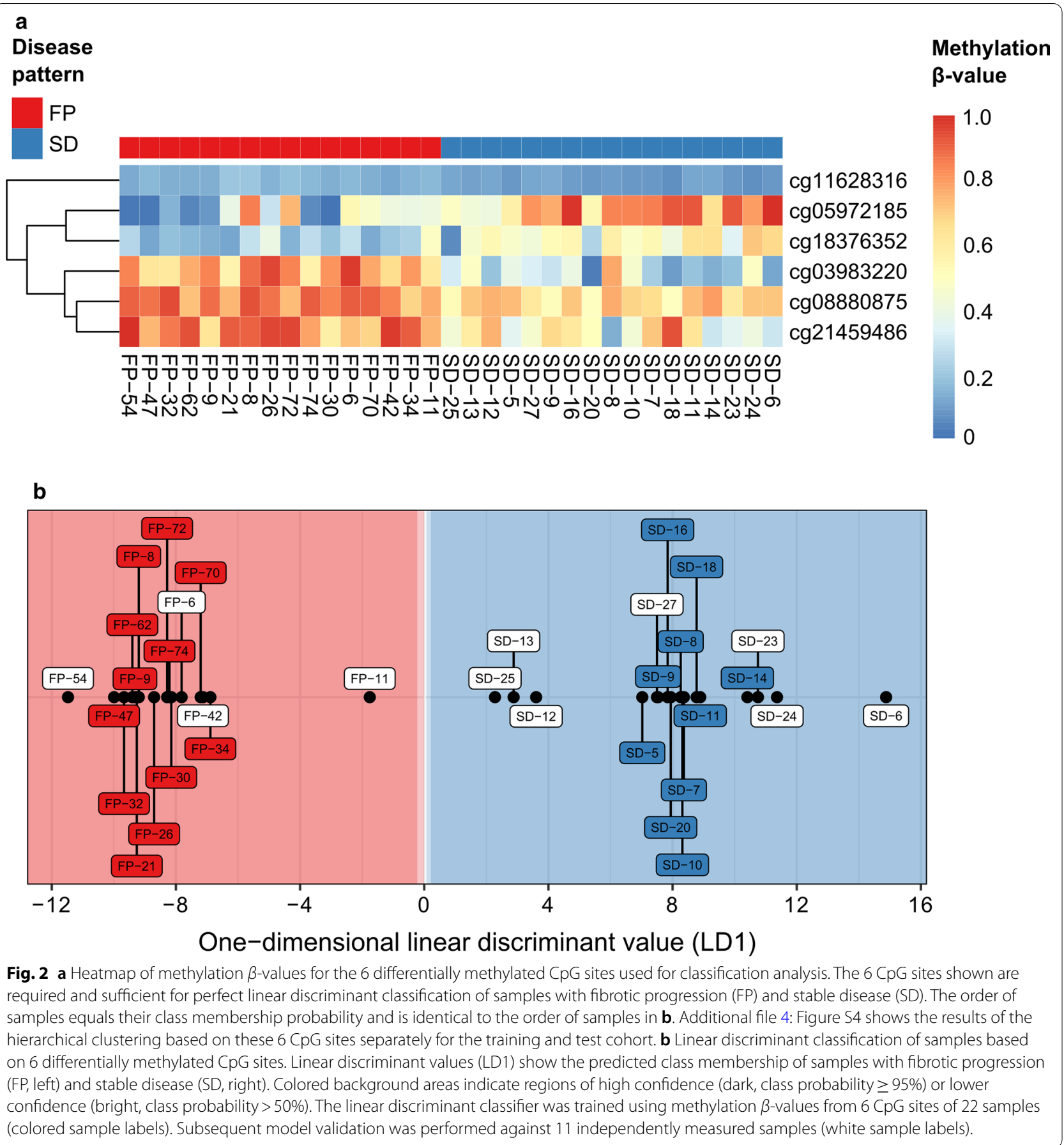

and in a more quantitative way (Additional file 10: Figure S8). However, hyper- and hypomethylation take place in both patient cohorts simultaneously in a complex way.

\section{Copy number alterations and fibrotic progress}

In a next step, we investigated whether copy number gains or losses are able to identify patients undergoing fibrotic progress and whether a CNA analysis might support the DNA methylation classifier. Calculating gene copy number alterations from the genome wide DNA methylation data obtained with the EPIC array is a straightforward well established procedure [24].

In Fig. 3a, the median copy number values for each chromosome in the group showing progression to myelofibrosis were calculated using the group with stable disease as a reference. No gains or losses of segments 
Table 1 Genomic localization and functional annotation of the 6 CpG sites shown in Fig. 2a

\begin{tabular}{llllll}
\hline Chr & Name & Localization & Associated gene & & Coefficient for LDM \\
\hline 16 & cg03983220 & OpenSea & None & & -7.431161 \\
10 & cg11628316 & Island & NM_001008541 & Max-interacting protein 1 & -44.910325 \\
2 & cg08880875 & N_Shore & NM_138804 & Meiosis 1 Associated Protein & -11.469252 \\
13 & cg21459486 & OpenSea & none & & -9.140643 \\
16 & cg05972185 & OpenSea & NM_001164766 & Zinc finger homeobox 3 & 6.017707 \\
19 & cg18376352 & Island & NM_004750 & Cytokine Receptor-Like Factor 1 & 12.946803 \\
\hline
\end{tabular}

For the definition and nomenclature of CpG islands, shores, open sea, and shelves, see $[49,50]$

LDM Linear discriminant model

(absolute $\log 2$ copy ratio $\geq 0.5$ ) capable of distinguishing the two sample sets could be detected.

\section{Mutation profiles and fibrotic progress}

In recent years, several groups [7, 8, 25] including our own [9] identified mutational signatures associated with fibrotic progress. Despite strong statistically significant correlations comparing groups of patients the predictive power of mutation profiling for individual samples can be improved, especially in the absence of these alterations.

Mutations in ASXL1, EZH2, IDH1/2, and, SRSF2, called "high molecular risk, HMR" [26] or "molecular high risk, MHR" [27] mutations, have been associated with worse prognosis in PMF patients. There is a clear statistical significant association of the presence of one or more mutation of this type with fibrotic progress also in our cohort: 7 out of 16 (44\%) with fibrotic progress versus 1 out of 20 (5\%) with stable disease, $\mathrm{Chi}^{2}$-test, $p=0.0065$. However, 7 out of 16 samples (44\%) from patients displaying progress to myelofibrosis do not have any mutation in one of these five genes (Fig. 3b), indicating the limitation of taking only gene mutations into account.

In contrast to the methylation profile, the presence of well-described high molecular risk mutations in ASXL1, $E Z H 2, I D H 1 / 2$, or $S R S F 2$ is not able to predict progression to myelofibrosis $(p=0.99$ in the multivariate analysis, see below).

\section{Multivariate analysis of clinical data and methylation scores}

For each patient, all available clinical parameters (patient age at diagnosis, patient sex, mutation status, leukocyte count, platelet count, and hemoglobin concentration) as well as the LDA value calculated from the methylation $\beta$-values of $6 \mathrm{CpG}$ sites were used for an explorative multivariate analysis. The numbers of leukocytes and platelets were only available for 18 out of 33 patients and the concentration of hemoglobin only for 17 out of
33 patients. Therefore, these parameters were modeled independently from the other parameters using Bayesian logistic regression.

All demographic and clinical data including age, sex, leukocyte count, platelet count, and hemoglobin concentration did not show any statistically significant difference between the two cohorts. Only rare CHIP mutations (Fig. 3b) were significant for regression $(p=0.0346)$. However, the LD value calculated for each sample showed a better level of significance in this analysis $(p=0.0098)$ and also allows for accurate classification of samples with fibrotic progression but without rare CHIP mutations (which are frequent but not ubiquitous in samples with fibrotic progression).

\section{Epigenetic aging and fibrotic progress}

It has already been speculated for a long time that DNA methylation aberrations accumulate over the life span of an organism due to the intrinsic error rates of the enzymatic machinery responsible for proper maintenance of these patterns (see [28] and references therein) and that these accumulating DNA methylation aberrations contribute to the development of various diseases including cancer $[11,12]$. Developing this idea further, several groups established algorithms based on the quantitative assessment of DNA methylation patterns to measure the epigenetic age (EA) of a given patient sample in comparison with its chronological age (CA) [29]. Discrepancies between the epigenetic and the chronological age might indicate increased risk for developing certain pathological conditions.

Three algorithms have been developed and are widely discussed in the literature: the Horvarth [30], Hannum [31], and PhenoAge [32] signature. In this study, we are not interested in measuring the chronological age of the samples (which is known and documented) by analyzing DNA methylation patterns. Instead, discrepancies between the (known) chronological age and the calculated "epigenetic" age are of interest in our context. These 
(See figure on next page.)

Fig. 3 a Copy number alterations (CNA) in cases with stable disease versus cases showing progression to myelofibrosis. Log2 copy ratios for individual $\mathrm{CpG}$ sites as well as segments were calculated from the median signal intensity of samples with fibrotic progression compared to samples with stable disease as reference set. The $x$-axis visualizes genomic locations with centromere positions indicated by dashed lines, whereas the $y$-axis indicates individual log2 copy ratios. No gains or losses of segments (absolute log2 copy ratio $\geq 0.5$ ) could be detected between both sample sets. $\mathbf{b}$ Results of the mutation analysis. The cases showing progress to myelofibrosis are represented in the upper part of the figure, the cases with stable disease in the lower part. Each red square indicates the presence of a mutation causing a loss of function or an altered protein function. The asterisk on the right side $\left(^{*}\right)$ mark the three samples which failed the initial QC procedure, but were classified correctly using the linear discriminant classifier (see Additional file 1: Figure S1). Since the case series of this study is a subset of the series described in detail before by us the results of the mutation profiling are also contained within Fig. 2 of Bartels et al.

differences might indicate premature aging of patients associated with morbidity. Therefore, PhenoAge is in our context the most suitable approach [29].

Figure 4a shows that in 9 out of 16 cases with fibrotic progress (65.3\%), the calculated epigenetic age is higher than the chronological age, whereas for the group with stable disease, this is the case in only 3 out of 17 cases (17.6\%, chi ${ }^{2}$-test, $\left.p=0.021\right)$ indicating an association between risk of progress to myelofibrosis and an increase in epigenetic age. Figure 4b confirms this relationship by calculating differences between mean chronological and mean epigenetic age of both patient groups (not by evaluating and counting individual cases as in Fig. 4a. The calculated epigenetic age in the cohort without progression to fibrosis (SD) is lower than the chronological age in this group, whereas in the cohort with fibrotic progression both are very similar, meaning that the mean calculated epigenetic age is on average higher in the patients who later develop myelofibrosis compared to the patients with stable disease ( $p=0.003$ Mann-Whitney- $U$ test).

\section{Discussion}

Analyzing histopathologically well characterized bone marrow trephines from prefibrotic PMF patients, we could show for the first time that the analysis of DNA methylation patterns is able to identify those patients who will progress to myelofibrosis. For this purpose, we employed the EPIC array technology which interrogates the methylation status of 850,000 individual CpG sites.

In comparison with other hematological malignancies, surprisingly little is known about DNA methylation patterns in MPNs, their dynamics during course of disease, and their diagnostic and prognostic impact. In two recent comprehensive reviews about myeloproliferative diseases, aberrations in DNA methylation are not mentioned at all $[25,33]$.

The review by Kim and Abdel-Wahab entitled "Focus on the epigenome in the myeloproliferative neoplasms" [34] concentrates nearly exclusively on mutations in genes encoding proteins involved in DNA and histone modifications and mentions alterations in these modifications itself (i.e., alterations in DNA methylation) only in passing.

Nischal et al. [35] demonstrated in their pioneering study using HELP assay (HpaII tiny fragment enriched by LM-PCR) that comprehensive DNA methylation profiling is able to distinguish the three $B C R-A B L 1$-negative MPN subgroups polycythemia vera $(n=8)$, essential thrombocytosis $(n=6)$, and primary myelofibrosis $(n=11)$. Samples from pre-fibrotic PMF patients later showing progression to myelofibrosis were not analyzed at that time.

The findings from Nischal et al. were confirmed by Perez et al. [16] using the $27 \mathrm{k}$ methylation array from Illumina. Due to the limited resolution of the technology they found mostly differences between (1) MPN samples and healthy controls and (2) MPN samples and MPN samples transformed into AML. Nielsen et al. [17] using the much more comprehensive $450 \mathrm{k}$ array from Illumina focused in their interesting DNA methylation profiling study on different cellular compartments of the hematopoietic system analyzing flow-sorted cells and the relationship between DNA methylation on the one hand and histone modifications and gene mutations on the other hand. The course of disease within individual patients (i.e., progression to myelofibrosis) was not under study.

The interesting association between ASXL1 mutations and distinct methylation profiles reported by Nielsen et al. [17] could not be confirmed in our study because both our groups included only a single $A S X L 1$ mutated case each. Also, DNMT3A and TET2 alterations are too rare in our cohort for robust meaningful conclusions. Only the previously reported association of mutations rarely affected by age-related clonal hematopoiesis $(\mathrm{ARCH})$ with progression to fibrosis [9] could be confirmed (see Fig. 4b). However, 6 from 16 patients (37.5\%) undergoing progression to myelofibrosis did not show any mutation commonly found in MDS and only rarely in ARCH. Four patients (25\%) did not show any mutation in the set of 25 genes tested, indicating certain limitations of this approach in analyzing individual cases. The multivariate analysis of all parameters under study clearly showed that the presence of so-called high risk mutations 


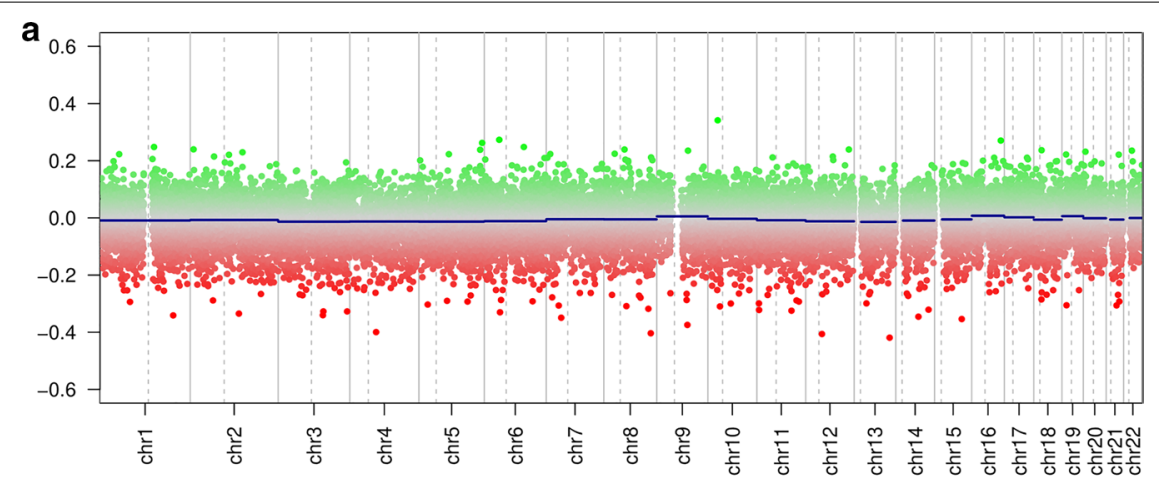

b

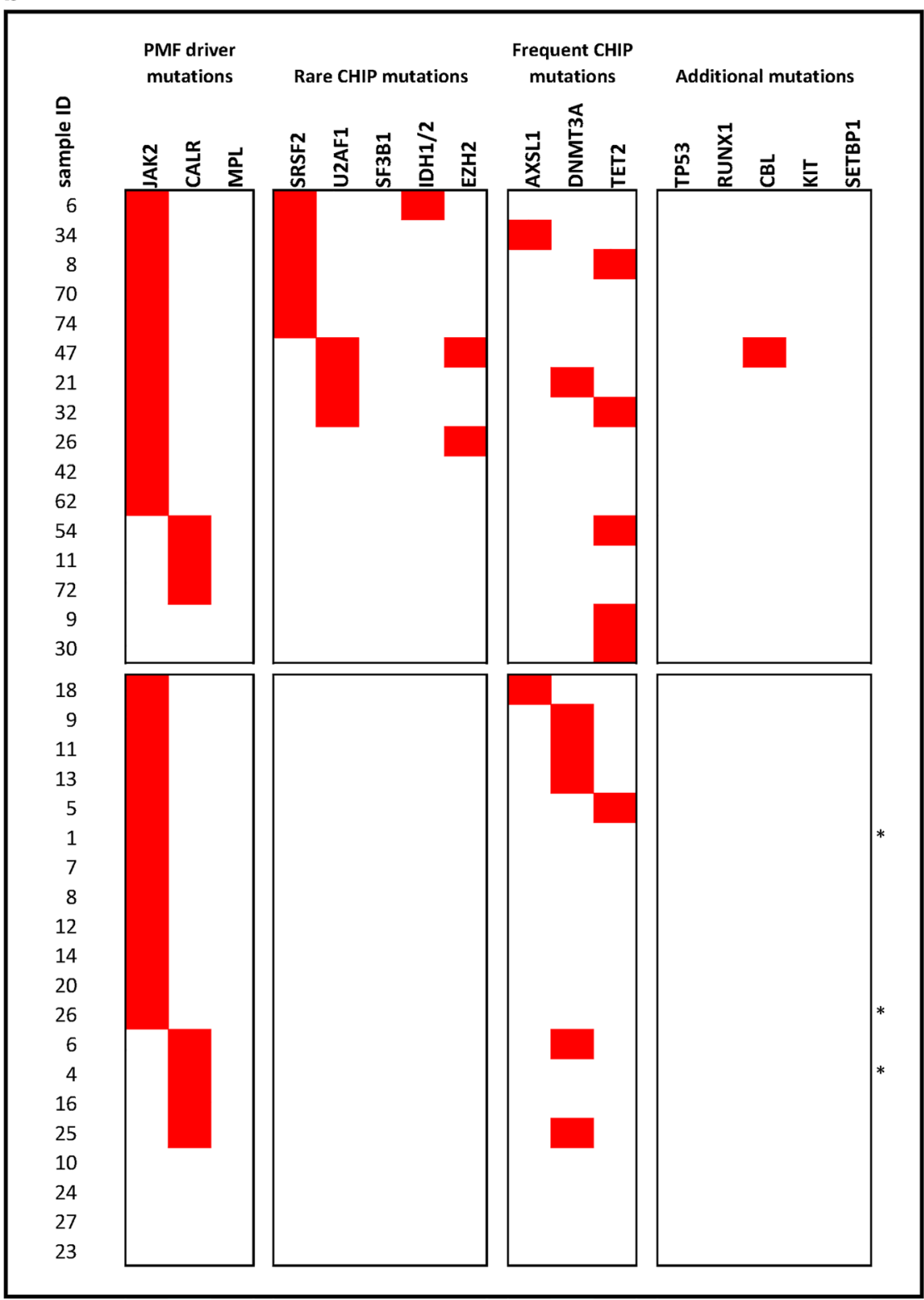



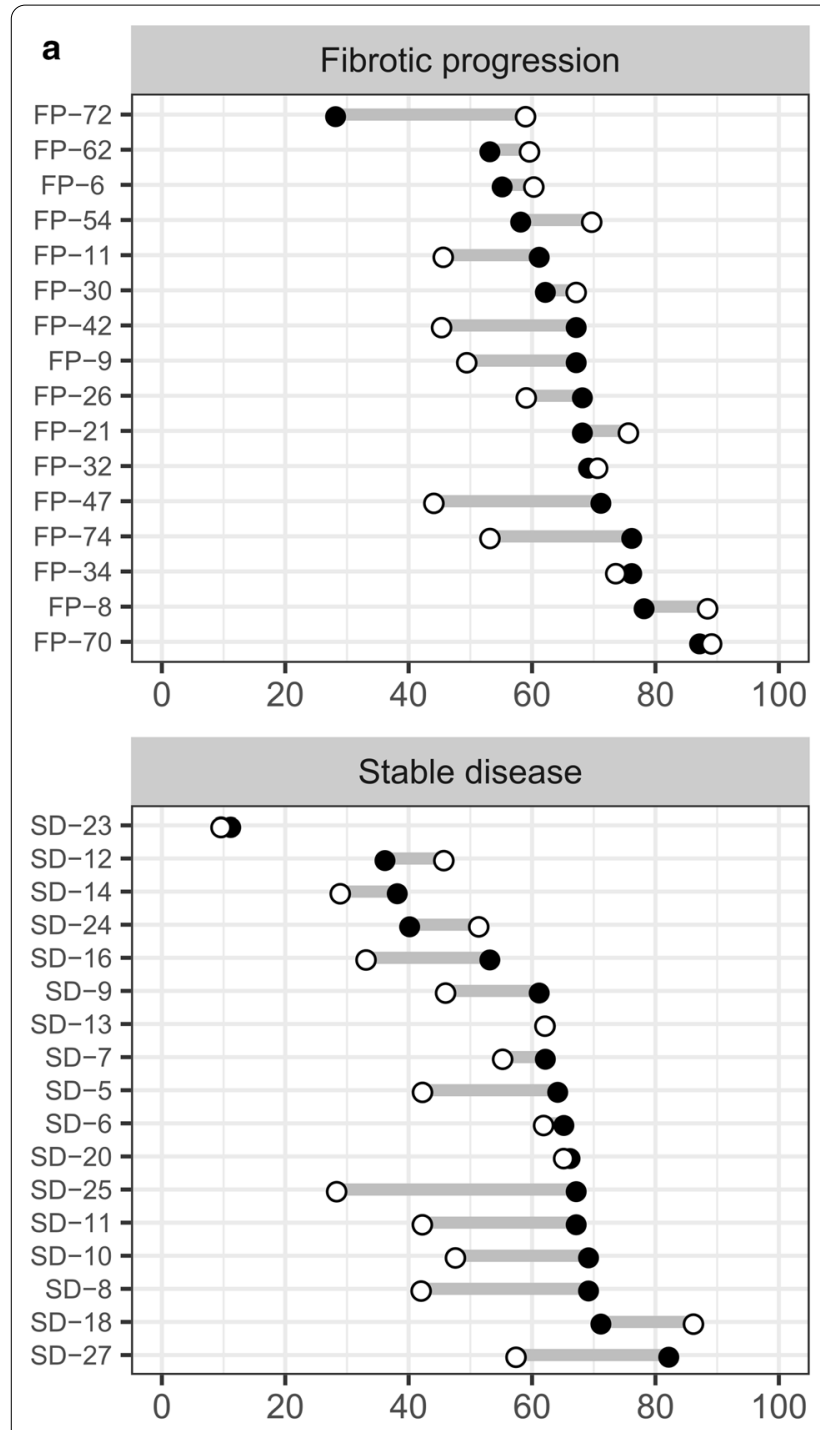

\section{- Chronologic age \\ OEpigenetic age (PhenoAge)}

Fig. 4 a Differences in epigenetic and chronological age per patient. Each line represents an individual patient. The calculated epigenetic age is larger than the chronological age in 9 out of 16 cases with fibrotic progress $(65,3 \%)$, in the group with stable disease this is the case in only 3 out of 17 cases $\left(17,6 \%\right.$, Chi ${ }^{2}$-test, $\left.p=0.021\right)$. b Differences in epigenetic and chronological age per disease group. Comparing the mean epigenetic age and the mean chronological age between both sample sets it turns out that the mean of the „PhenoAge "is statistically significantly higher in the group showing fibrotic progression (FP) compared to the stable disease (SD) group ( $p=0.003$ Mann-Whitney- $U$ test), whereas the mean chronological age is very similar in both disease groups

in $A S X L 1, E Z H 2, I D H 1 / 2$, or SRSF2 is not able to predict progression to myelofibrosis $(p=0.99)$.

Also mutations in the tumor suppressor gene TP53, incorporated by Grinfeld et al. in their recently published "MPN Personal Risk Score"[36], do not contribute to a separation of the two patient groups in our study, because they are too rare in our prefibrotic PMF cases.

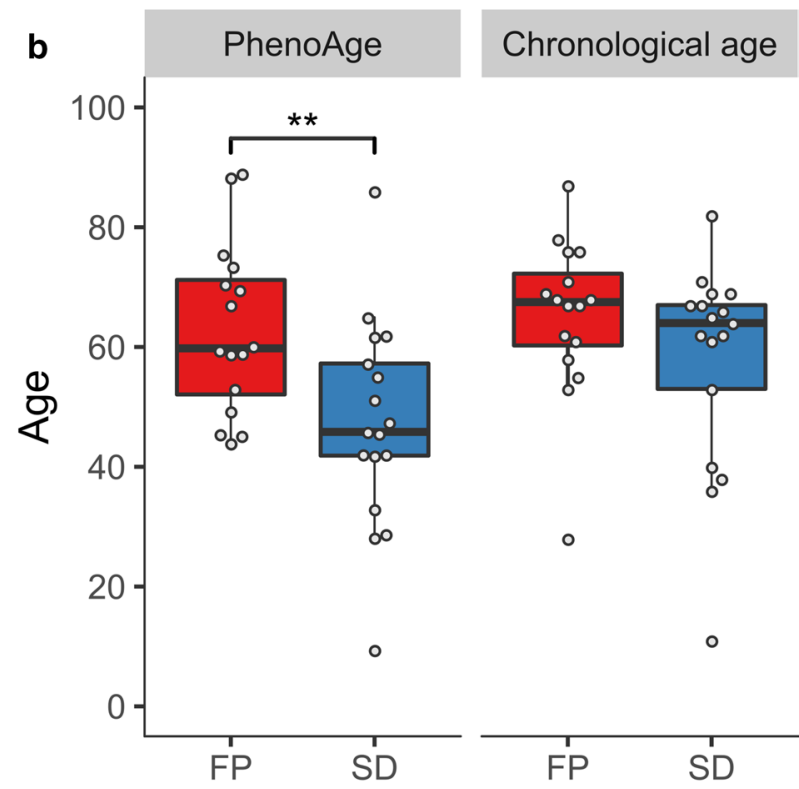


In a more recent genome-wide DNA methylation profiling project (also using Illumina's $450 \mathrm{~K}$ array), Martinez-Calle et al. [18] identified only differences between patients with myelofibrosis and healthy controls, similar to the findings reported earlier by Perez et al. [16]. No differences within the group of patients with myelofibrosis were reported, and the question of fibrotic progress has also not been addressed in this study.

Comparing DNA methylation profiles from healthy controls and patients with a certain disease or from patients with different diseases always comes with the risk of confounding factors distorting the DNA methylation profiles, the most important being cellular composition of the blood or the bone marrow, respectively (see [37] and references therein). Therefore, we compared only DNA methylation profiles from patients with the same disease and disease stage, i.e., prefibrotic PMF (MF0 and EB0). The only difference between the two groups is the later course of disease (i.e., with and without progression to myelofibrosis). This focus on patients diagnosed with prefibrotic PMF (EB0, MF0) avoids all confounding factors distorting the DNA methylation profiles.

The influence of the epigenetic age on the course of disease in the context of myeloproliferative disease has also been studied by McPerson et al.[38]. However, they analyzed only patients with polycythaemia vera (PV) and essential thrombocythaemia (ET), before and after treatment with the histone deacetylase inhibitor vorinostat, finding opposite effects in both diseases. They used the three-gene aging signature proposed by Weidner et al. [39] for analyzing changes in DNA methylation during treatment. Therefore, the interesting results of this study and the results presented here cannot be compared directly.

The gene ontology analysis identified biological processes and molecular functions related to cell-cell and cell-matrix adhesion as highly enriched in the group of differentially methylated genes (see Additional file 5: Table S1), indicating that these processes might be more important for disease progression than, e.g., proliferation or cell death (apoptosis). In other organs, like liver or lung, the role of adhesion molecules for the development of fibrosis is already well studied [40, 41]. Future functional studies have to elucidate the role of the differentially methylated regions identified herein for the progression to myelofibrosis in PMF patients.

A limitation of the present study is the modest sample size which should be increased in follow-up studies. Multi-center collaborations will be necessary to collect much larger numbers of appropriate samples with complete clinical and histopathological records.
Table 2 Clinical parameters

\begin{tabular}{lll}
\hline & $\begin{array}{l}\text { Progression } \\
\text { to fibrosis (FP) } \\
\end{array}$ & Stable disease (SD) \\
& $\mathbf{n = 1 6}$ & $\mathbf{n = 2 0}$ \\
\hline Females & $6(38 \%)$ & $11(55 \%)$ \\
Age (at diagnosis, mean) & 62 years & 56 years \\
Total follow-up & 75.5 years & 154 years \\
Range & $1.0-12.5$ years & $4.0-14.0$ years \\
Mean follow-up & 5 years & 7.3 years \\
Hemoglobin $(\mathrm{g} / \mathrm{dL}, \mathrm{median})$ & 11.7 & 14.8 \\
Leukocytes $\left(\times 10^{6} \times \mathrm{L}^{-1}\right)$ & 16.5 & 9.2 \\
Thrombocytes $\left(\times 10^{9} \times \mathrm{L}^{-1}\right)$ & 776 & 912 \\
Spleen size $(\mathrm{mean})$ & $13.4 \mathrm{~cm}$ & 14.3 \\
\hline
\end{tabular}

\section{Conclusions}

This study compares, to the best of our knowledge, for the first time the DNA methylation profile in pre-fibrotic PMF patients who later develop overt myelofibrosis with the DNA methylation profile from pre-fibrotic PMF patients not progressing to myelofibrosis. This approach identifies the prognostic potential of comprehensive DNA methylation profiling for patients presenting with prefibrotic PMF. In addition, the importance of cell-cell and cell-matrix interactions for these processes is highlighted by the enrichment in the gene ontology analysis and should be followed-up in future studies.

The technique used in this study is already well established in other diagnostic settings (see [24, 42-44] as examples) and should be implementable with reasonable efforts after independent validation in a larger cohort.

\section{Materials and methods \\ Patient samples}

Decalcified formalin-fixed and paraffin-embedded bone marrow trephines collected between 2000 and 2018 were retrieved retrospectively from the archive of the Institute of Pathology, Hannover Medical School. All cases underwent histopathological routine diagnostic procedures. For the cohort displaying progression to fibrosis (FP), cases with two consecutive biopsies and a minimum of 1 year follow-up time (range: $1-12.5$ years, mean: 4 years, median: 2 years) were selected. The first bone marrow biopsy at the time of initial diagnosis had to be classified as prefibrotic PMF without increase in blasts (EB0) according to WHO definition [45] and with no signs of myelofibrosis (MF0). The second biopsy showed clear progression to myelofibrosis (MF 2 or 3). For the stable disease (SD) cohort without development of fibrosis, a minimum of 4 years follow-up time (range: 4-14 years, mean: 7.3 years, median: 6 years) was required with 
clinically and histologically persisting prefibrotic PMF. The first and last biopsies in this cohort were MF grade 0 and EB grade 0 . For further details, see Table 2. In this cohort without progression to fibrosis, the reasons for performing subsequent bone-marrow biopsies were quite heterogeneous, e.g., anemia or thrombosis. (for details see ref [9]).

The cohort analyzed in this study represents a subgroup of the cohort described in detail in Bartels et al. 2020 [9]. The selection criteria for the training set and the validation set were the availability of a sufficient amount of DNA (therefore, not all samples from Bartels et al. could be included in this study) and a balanced ratio between cases with stable disease and progression to fibrosis, respectively.

The study design is following the guidelines of the Hannover Medical School ethics committee for retrospective analyses.

\section{DNA extraction}

Extraction of genomic DNA was performed using the Maxwell RSC instrument (Promega, Madison, WI, USA) and the Maxwell RSC DNA FFPE kit according to the manufacturer's instructions. Three to five sections of $10 \mu \mathrm{m}$ thickness each were taken, depending on the size of the trephine. After extraction, nucleic acid concentrations were quantified using a Qubit 2.0 fluorimeter (ThermoFisherScientific, Carlsbad, CA, USA) and the Qubit dsDNA high sensitivity kit (ThermoFisherScientific, Carlsbad, CA, USA).

\section{DNA methylation analysis}

For genome-wide DNA methylation analyses, the $850 \mathrm{k}$ EPIC array platform from Illumina was used [46]. At least $250 \mathrm{ng}$ genomic DNA extracted from FFPE bone marrow trephines was used. In the majority of cases, 500 ng genomic DNA were available. Bisulfite conversion, array hybridization, and raw data collection were done at Life\&Brain (Bonn, Germany) following the manufacturer's protocols. Subsequently, raw data files were downloaded from this company's server for further analyses. The $850 \mathrm{~K}$ EPIC array data series are deposited in the Gene Expression Omnibus (GEO) data base (GEO accession number: GSE152519). Array data analysis was performed with $\mathrm{R}$ (version 3.4.4). The $\mathrm{R}$ package IlluminaHumanMethylationEPICanno.ilm10b3.hg19 (version 0.6.0) was used for chip annotation. The $\mathrm{R}$ package MethylAid (version 1.12.0) was used for quality control (default settings) [47]. The $\mathrm{R}$ package ChAMP (version 2.9.10) was used for data loading, sample exclusion, and batch effect correction (all default settings) as well statistical analyses and intra-sample probe normalization via the BMIQ algorithm $[48,49]$. The batch variable
"AMP_Plate" was designated as confounder and the variable "Sample_Class" as predictor for batch correction via the ComBat algorithm [50]. No batch correction was performed on data used for machine learning and modeling. Differentially methylated probes (DMPs, representing individual CpG sites) were identified using the limma algorithm included in the ChAMP package with a false discovery rate (FDR) threshold of $\leq 0.1$ [51]. Therefore, in this study the definition of "differentially methylated" is solely driven by statistical analyses (via the FDR) and not by a $\beta$-value threshold. Gene set enrichment analysis (GSEA) against the Gene Ontology (GO) database was performed based on the differentially methylated regions (DMR) between both groups, which were identified using the Bumphunter algorithm included in the ChAMP package with an FDR of $\leq 0.05$ and the parameters 'maxGap' and 'cutoff' changed from their default values to 250 and 0.99 , respectively. The GSEA itself was performed using the gometh algorithm included in the ChAMP package and an FDR threshold of 0.1. The R package "conumee" in bioconductor was employed for identification of copy number variations [52]. For the calculation of the epigenetic methylation age, the PhenoAge algorithm from the R package ENmix (version 1.25.1) was used [32, 53].

\section{Classification of samples}

In order to select suitable DMPs for sample classification, we utilized an exact leaps and bounds algorithm aimed at optimizing the Tau-squared coefficient from the $\mathrm{R}$ package sub-select (version 0.14 [54]). The resulting $\mathrm{CpG}$ subsets were used to create data sets from the training set of 22 samples as input for the implementation of linear discriminant analysis (LDA) in the R package caret (version 6.0-81 [23]). It is important to point out that in contrast to the data used for statistical analysis; no batch correction was performed on data used for classification of samples. A singular value decomposition analysis shows that ComBat batch correction could be successfully used to reduce the influence of confounding variables (such as AMP_Plate) on the methylation data set (see Additional file 11: Figure S9). For resampling and estimating preliminary model performance, we used fivefold repeated cross-validation. Tuning parameters were left at default settings. All models were finally evaluated concerning their accuracy against a validation set of 11 independently measured samples. When yielding the same accuracy, models were preferred that require less input features (i.e., DMPs). 


\section{Multivariate analysis}

In order to deal with the moderate sample sizes and occasional separation by chance, we used Bayesian logistic regression instead of logistic regression for our analysis of demographic and clinical data. Parameters were considered to be significant if their $p$-value for the $Z$-statistic was below 0.05 .

\section{Mutation profiling}

Targeted re-sequencing of 23 genes was performed using an amplicon-based NGS panel, and pyrosequencing was used for analysis of MPL Codon 515 and for ETNK1 Codon 244 as described [55].

\section{Analysis of copy number alterations}

The analysis of copy number variations based on their methylation profiles was performed using the $\mathrm{R}$ package conumee (version 1.12.0) [52]. For this purpose, the signal intensities of individual samples with fibrotic progression as well as the median signal intensity of these samples was compared to the set of samples with stable disease as a reference (default settings).

\section{Supplementary Information}

The online version contains supplementary material available at https:/doi. org/10.1186/s13148-021-01010-y.

Additional file 1: Figure S1. Singular value decomposition analysis for identification and exclusion of confounding factors. Sample age and amount of DNA both show a $p$-value above 0.05 in the SVD analysis, demonstrating that these two variables are clearly not confounding factors.

Additional file 2: Figure S2. Hierarchical clustering based on $25 \mathrm{CpG}$ sites from Fig. 1 separately for the training and test cohort.

Additional file 3: Figure S3. Hierarchical clustering based on 1000 differentially methylated $\mathrm{CpG}$ sites and histogram of $\beta$-values. This figure corresponds to Fig. 1. The lower panel shows the histogram for all $\beta$-values illustrating the gain in methylation in the fibrotic progression cohort, accompanied also by a loss of DNA methylation in a smaller group of CpG sites.

Additional file 4: Figure S4. Hierarchical clustering based on $6 \mathrm{CpG}$ sites from Fig. 2 separately for the training and test cohort.

Additional file 5: Table S1. Gene set enrichment analysis of differentially methylated regions against the Gene Ontology database.

Additional file 6: Table S2. Detailed description of the 40 most differentially methylated regions (DMR).

Additional file 7: Figure S5. Linear discriminant classification of lowquality samples. Three samples (white background labels) which had been excluded from the statistical analysis due to high proportions of probes above the detection $p$-value threshold of 0.1 are classified correctly using the linear discriminant model.

Additional file 8: Figure S6. Histogram of all $\beta$-values with fixed thresholds for hyper- and hypomethylation. With a $\beta$-value threshold of $>0.8$ for hypermethylation and $<0.1$ for hypomethylation the simultaneous gain in hyper- and in hypomethylated loci in the fibrotic progression cohort is obvious. The number of CpG sites with very high methylation level (i.e., above 0.8$)$ is larger in the FP cohort compared to the SD cohort $(245,829$ vs 204,494 , or $20.2 \%$ more highly methylated sites in the FP cohort) and this increase in highly methylated sites in the FP cohort is three times higher than the increase in CpG sites with very low methylation (i.e., below 0.1 ) in this cohort (relative to the SD cohort the number of CpG sites with low methylation level increases by only 7.7\%). Therefore, it is justified to state that overall the fibrotic progression cohort is characterized by hypermethylation relative to the stable disease cohort.

Additional file 9: Figure S7. Boxplots for the $\beta$-values of the 25 differentially methylated $\mathrm{CpG}$ sites shown in Fig. 1. The display of the individual boxplots shows the simultaneous gain and loss of DNA methylation in the fibrotic progression cohort relative to the stable disease cohort (as shown in a more global view in Additional file 8: Figure S6). 18 from 25 CpG sites display a higher methylation level in the FP group compared to the SD group.

Additional file 10: Figure S8. Number of differentially methylated CpG sites in the FP group versus the SD group. a Number of CpG sites more heavily methylated ("hypermethylated") in the FP or the SD group at a given threshold for the difference in the $\beta$-value. $\mathbf{b}$ Histogram for $\Delta \beta>0.1$. c Histogram for $\Delta \beta>0.2$. The predominance of more heavily methylated loci in the FP group is obvious. The SD group shows a distribution centered around $\beta$-values between 0.5 and 0.6 , whereas the FP group shows a clear skewing of the distribution towards $\beta$-values between 0.8 and 0.9 .

Additional file 11: Figure S9. Singular value decomposition analysis of the batch correction. ComBat batch correction could be successfully used to reduce the influence of confounding variables (such as AMP_Plate) on the methylation data set, leaving "sample class" as the most important discriminator.

Additional file 12: Table S3. Detailed description of the 25 most differentially methylated $\mathrm{CPG}$ sites at FDR $<0.1$.

\section{Authors' contributions}

$\mathrm{UL}, \mathrm{HS}, \mathrm{SB}$, and HK conceived the study, interpreted the data, and wrote the paper; GB, JS, and HK contributed the histopathological diagnosis and clinical data of the cases. All authors approved the final version of the manuscript.

\section{Funding}

Open Access funding enabled and organized by Projekt DEAL. This study was supported by intramural funding.

\section{Availability of data and materials}

The DNA methylation array data are deposited at GEO (GSE152519).

\section{Ethics approval and consent for publication}

The study design is following the guidelines of the Hannover Medical School ethics committee for retrospective analyses of anonymized samples. All authors have read the manuscript in its final form and approved submission.

\section{Competing interests}

The authors declare that they have no competing interests.

Received: 25 September 2020 Accepted: 11 January 2021

Published online: 04 February 2021

\section{References}

1. Nangalia J, Green AR. Myeloproliferative neoplasms: from origins to outcomes. Blood. 2017;130:2475-83.

2. Vainchenker W, Kralovics R. Genetic basis and molecular pathophysiology of classical myeloproliferative neoplasms. Blood. 2017:129:667-79.

3. Latagliata R, Polverelli N, Tieghi A, Palumbo GA, Breccia M, Sabattini E, Villari L, Riminucci M, Valli R, Catani L, et al. Comparison of JAK2(V617F) -positive essential thrombocythaemia and early primary myelofibrosis: the impact of mutation burden and histology. Hematol Oncol. 2018:36:269-75.

4. Kroger N, Giorgino T, Scott BL, Ditschkowski M, Alchalby H, Cervantes F, Vannucchi A, Cazzola M, Morra E, Zabelina T, et al. Impact of allogeneic 
stem cell transplantation on survival of patients less than 65 years of age with primary myelofibrosis. Blood. 2015;125:3347-50.

5. Kroger NM, Deeg JH, Olavarria E, Niederwieser D, Bacigalupo A, Barbui T, Rambaldi A, Mesa R, Tefferi A, Griesshammer M, et al. Indication and management of allogeneic stem cell transplantation in primary myelofibrosis: a consensus process by an EBMT/ELN international working group. Leukemia. 2015;29:2126-33.

6. Guglielmelli P, Lasho TL, Rotunno G, Mudireddy M, Mannarelli C, Nicolosi M, Pacilli A, Pardanani A, Rumi E, Rosti V, et al. MIPSS70: mutationenhanced international prognostic score system for transplantation-age patients with primary myelofibrosis. J Clin Oncol. 2018;36:310-8.

7. Tefferi A, Guglielmelli P, Lasho TL, Gangat N, Ketterling RP, Pardanani A, Vannucchi AM. MIPSS70+ Version 2.0: mutation and karyotype-enhanced international prognostic scoring system for primary myelofibrosis. J Clin Oncol. 2018;36:1769-70.

8. Tefferi A, Guglielmelli P, Nicolosi M, Mannelli F, Mudireddy M, Bartalucci N, Finke CM, Lasho TL, Hanson CA, Ketterling RP, et al. GIPSS: genetically inspired prognostic scoring system for primary myelofibrosis. Leukemia. 2018;32:1631-42.

9. Bartels S, Faisal M, Busche G, Schlue J, Hasemeier B, Schipper E, Vogtmann J, Westphal L, Lehmann U, Kreipe H. Mutations associated with agerelated clonal hematopoiesis in PMF patients with rapid progression to myelofibrosis. Leukemia. 2019;34:1364-72.

10. Beerman I, Bock C, Garrison BS, Smith ZD, Gu H, Meissner A, Rossi DJ. Proliferation-dependent alterations of the DNA methylation landscape underlie hematopoietic stem cell aging. Cell Stem Cell. 2013;12:413-25.

11. Dor Y, Cedar H. Principles of DNA methylation and their implications for biology and medicine. Lancet. 2018;392:777-86.

12. Ehrlich M. DNA hypermethylation in disease: mechanisms and clinical relevance. Epigenetics. 2019;14:1141-63.

13. Fourouclas N, Li J, Gillby DC, Campbell PJ, Beer PA, Boyd EM, Goodeve AC, Bareford D, Harrison CN, Reilly JT, et al. Methylation of the suppressor of cytokine signaling 3 gene (SOCS3) in myeloproliferative disorders. Haematologica. 2008;93:1635-44.

14. Augello C, Gianelli U, Falcone R, Tabano S, Savi F, Bonaparte E, Ciboddo M, Paganini L, Parafioriti A, Ricca D, et al. PDGFB hypomethylation is a favourable prognostic biomarker in primary myelofibrosis. Leuk Res. 2015:39:236-41.

15. Augello C, Cattaneo D, Bucelli C, Terrasi A, Fermo E, Martinelli I, lurlo A, Gianelli U. CD18 promoter methylation is associated with a higher risk of thrombotic complications in primary myelofibrosis. Ann Hematol. 2016:95:1965-9.

16. Perez C, Pascual M, Martin-Subero Jl, Bellosillo B, Segura V, Delabesse E, Alvarez S, Larrayoz MJ, Rifon J, Cigudosa JC, et al. Aberrant DNA methylation profile of chronic and transformed classic Philadelphia-negative myeloproliferative neoplasms. Haematologica. 2013;98:1414-20.

17. Nielsen HM, Andersen CL, Westman M, Kristensen LS, Asmar F, Kruse TA, Thomassen M, Larsen TS, Skov V, Hansen LL, et al. Epigenetic changes in myelofibrosis: Distinct methylation changes in the myeloid compartments and in cases with ASXL1 mutations. Sci Rep. 2017;7:6774.

18. Martinez-Calle N, Pascual M, Ordonez R, Eneriz ESJ, Kulis M, Miranda E, Guruceaga E, Segura V, Larrayoz MJ, Bellosillo B, et al. Epigenomic profiling of myelofibrosis reveals widespread DNA methylation changes in enhancer elements and ZFP36L1 as a potential tumor suppressor gene that is epigenetically regulated. Haematologica. 2019;104:1572-9.

19. Kling T, Wenger A, Beck S, Caren H. Validation of the MethylationEPIC BeadChip for fresh-frozen and formalin-fixed paraffin-embedded tumours. Clin Epigenetics. 2017;9:33.

20. Ohara K, Arai E, Takahashi Y, Fukamachi Y, Ito N, Maeshima AM, Fujimoto $H$, Yoshida T, Kanai Y. Feasibility of methylome analysis using small amounts of genomic DNA from formalin-fixed paraffin-embedded tissue. Pathol Int. 2018;68:633-5.

21. Braczynski AK, Capper D, Jones DTW, Schittenhelm J, Stichel D, von Deimling A, Harter PN, Mittelbronn M. High density DNA methylation array is a reliable alternative for PCR-based analysis of the MGMT promoter methylation status in glioblastoma. Pathol Res Pract. 2020;216:152728.

22. Johnson WE, Li C, Rabinovic A. Adjusting batch effects in microarray expression data using empirical Bayes methods. Biostatistics. 2007;8:118-27.

23. Kuhn M, Wing J, Weston S, Williams A, Keefer C, Engelhardt A, Cooper T, Mayer Z, Kenkel B, Team RC, et al. caret: Classification adn Regression
Training. R package version 6.0-79. https://CRAN.R-project.org/packa ge $=$ caret. 2018.

24. Capper D, Stichel D, Sahm F, Jones DTW, Schrimpf D, Sill M, Schmid S, Hovestadt V, Reuss DE, Koelsche C, et al. Practical implementation of DNA methylation and copy-number-based CNS tumor diagnostics: the Heidelberg experience. Acta Neuropathol. 2018;136:181-210.

25. Gangat N, Tefferi A. Myelofibrosis biology and contemporary management. Br J Haematol. 2020;191:152-70.

26. Tefferi A, Guglielmelli P, Pardanani A, Vannucchi AM. Myelofibrosis Treatment algorithm 2018. Blood Cancer J. 2018;8:72.

27. Santos FPS, Getta B, Masarova L, Famulare C, Schulman J, Datoguia TS, Puga RD, Alves Paiva RM, Arcila ME, Hamerschlak N, et al. Prognostic impact of RAS-pathway mutations in patients with myelofibrosis. Leukemia. 2020;34:799-810.

28. Braga DL, Mousovich-Neto F, Tonon-da-Silva G, Salgueiro WG, Mori MA. Epigenetic changes during ageing and their underlying mechanisms. Biogerontology. 2020;21:423-43.

29. Field AE, Robertson NA, Wang T, Havas A, Ideker T, Adams PD. DNA methylation clocks in aging: categories, causes, and consequences. Mol Cell. 2018;71:882-95.

30. Horvath S. DNA methylation age of human tissues and cell types. Genome Biol. 2013;14:R115.

31. Hannum G, Guinney J, Zhao L, Zhang L, Hughes G, Sadda S, Klotzle B, Bibikova M, Fan JB, Gao Y, et al. Genome-wide methylation profiles reveal quantitative views of human aging rates. Mol Cell. 2013;49:359-67.

32. Levine ME, Lu AT, Quach A, Chen BH, Assimes TL, Bandinelli S, Hou L, Baccarelli AA, Stewart JD, Li Y, et al. An epigenetic biomarker of aging for lifespan and healthspan. Aging. 2018;10:573-91.

33. Schieber M, Crispino JD, Stein B. Myelofibrosis in 2019: moving beyond JAK2 inhibition. Blood Cancer J. 2019;9:74.

34. Kim E, Abdel-Wahab O. Focus on the epigenome in the myeloproliferative neoplasms. Hematol Am Soc Hematol Educ Program. 2013;2013:538-44.

35. Nischal S, Bhattacharyya S, Christopeit M, Yu Y, Zhou L, Bhagat TD, Sohal $D$, Will B, Mo Y, Suzuki M, et al. Methylome profiling reveals distinct alterations in phenotypic and mutational subgroups of myeloproliferative neoplasms. Cancer Res. 2013;73:1076-85.

36. Grinfeld J, Nangalia J, Baxter EJ, Wedge DC, Angelopoulos N, Cantrill R, Godfrey AL, Papaemmanuil E, Gundem G, MacLean C, et al. Classification and personalized prognosis in myeloproliferative neoplasms. N Engl J Med. 2018;379:1416-30.

37. Houseman EA, Kim S, Kelsey KT, Wiencke JK. DNA methylation in whole blood: uses and challenges. Curr Environ Health Rep. 2015;2:145-54.

38. McPherson S, Greenfield G, Andersen C, Grinfeld J, Hasselbalch HC, Nangalia J, Mills Kl, McMullin MF. Methylation age as a correlate for allele burden, disease status, and clinical response in myeloproliferative neoplasm patients treated with vorinostat. Exp Hematol. 2019;79:26-34.

39. Weidner Cl, Lin Q, Koch CM, Eisele L, Beier F, Ziegler P, Bauerschlag DO, Jockel KH, Erbel R, Muhleisen TW, et al. Aging of blood can be tracked by DNA methylation changes at just three $\mathrm{CpG}$ sites. Genome Biol. 2014:15:R24.

40. Agarwal SK. Integrins and cadherins as therapeutic targets in fibrosis. Front Pharmacol. 2014:5:131.

41. Hintermann E, Christen U. The many roles of cell adhesion molecules in hepatic fibrosis. Cells. 2019;8:1503.

42. Koelsche C, Hartmann W, Schrimpf D, Stichel D, Jabar S, Ranft A, Reuss DE, Sahm F, Jones DTW, Bewerunge-Hudler M, et al. Array-based DNAmethylation profiling in sarcomas with small blue round cell histology provides valuable diagnostic information. Mod Pathol. 2018;31:1246-56.

43. Benhamida JK, Hechtman JF, Nafa K, Villafania L, Sadowska J, Wang J, Wong D, Zehir A, Zhang L, Bale T, et al. Reliable clinical MLH1 promoter hypermethylation assessment using a high-throughput genome-wide methylation array platform. J Mol Diagn. 2020;22:368-75.

44. Perez E, Capper D. Invited review: DNA methylation-based classification of paediatric brain tumours. Neuropathol Appl Neurobiol. 2020;46:28-47.

45. Arber DA, Orazi A, Hasserjian R, Thiele J, Borowitz MJ, Le Beau MM, Bloomfield CD, Cazzola M, Vardiman JW. The 2016 revision to the World Health Organization classification of myeloid neoplasms and acute leukemia. Blood. 2016;127:2391-405.

46. Pidsley R, Zotenko E, Peters TJ, Lawrence MG, Risbridger GP, Molloy P, Van Djik S, Muhlhausler B, Stirzaker C, Clark SJ. Critical evaluation of the 
Illumina MethylationEPIC BeadChip microarray for whole-genome DNA methylation profiling. Genome Biol. 2016;17:208.

47. van Iterson M, Tobi EW, Slieker RC, den Hollander W, Luijk R, Slagboom $P E$, Heijmans BT. MethylAid: visual and interactive quality control of large Illumina 450k datasets. Bioinformatics. 2014;30:3435-7.

48. Teschendorff AE, Marabita F, Lechner M, Bartlett T, Tegner J, GomezCabrero D, Beck S. A beta-mixture quantile normalization method for correcting probe design bias in Illumina Infinium 450 k DNA methylation data. Bioinformatics. 2013;29:189-96.

49. Tian Y, Morris TJ, Webster AP, Yang Z, Beck S, Feber A, Teschendorff AE. ChAMP: updated methylation analysis pipeline for Illumina BeadChips. Bioinformatics. 2017:33:3982-4.

50. Luo J, Schumacher M, Scherer A, Sanoudou D, Megherbi D, Davison T, Shi T, Tong W, Shi L, Hong H, et al. A comparison of batch effect removal methods for enhancement of prediction performance using MAQC-II microarray gene expression data. Pharmacogenomics J. 2010;10:278-91.

51. Ritchie ME, Phipson B, Wu D, Hu Y, Law CW, Shi W, Smyth GK. limma powers differential expression analyses for RNA-sequencing and microarray studies. Nucleic Acids Res. 2015;43:e47.
52. Hovestadt V, Zapatka M. Conumee: enhanced copy-number variation analysis using Illumina DNA methylation arrays. R package version 1.9.0 http://bioconductor.org/packages/conumee/.

53. Xu Z, Niu L, Li L, Taylor JA. ENmix: a novel background correction method for Illumina HumanMethylation450 BeadChip. Nucleic Acids Res. 2016;44:e20.

54. Cerdeira JO, Silva PD, Cadima J, Minhoto M. Subselect: Selecting Variable Subsets. R package version 0.14. https://CRAN.R-project.org/packa ge=subselect. 2018.

55. Faisal M, Stark H, Busche G, Schlue J, Teiken K, Kreipe HH, Lehmann U, Bartels $\mathrm{S}$. Comprehensive mutation profiling and mRNA expression analysis in atypical chronic myeloid leukemia in comparison with chronic myelomonocytic leukemia. Cancer Med. 2019;8(2):742-50.

\section{Publisher's Note}

Springer Nature remains neutral with regard to jurisdictional claims in published maps and institutional affiliations.
Ready to submit your research? Choose BMC and benefit from:

- fast, convenient online submission

- thorough peer review by experienced researchers in your field

- rapid publication on acceptance

- support for research data, including large and complex data types

- gold Open Access which fosters wider collaboration and increased citations

- maximum visibility for your research: over $100 \mathrm{M}$ website views per year

At BMC, research is always in progress.

Learn more biomedcentral.com/submissions 